\title{
Correlations between Cortical and Locomotor Muscle in the Seven Gait Phases during Treadmill Walking
}

\section{CURRENT STATUS: POSTED}

Research Square

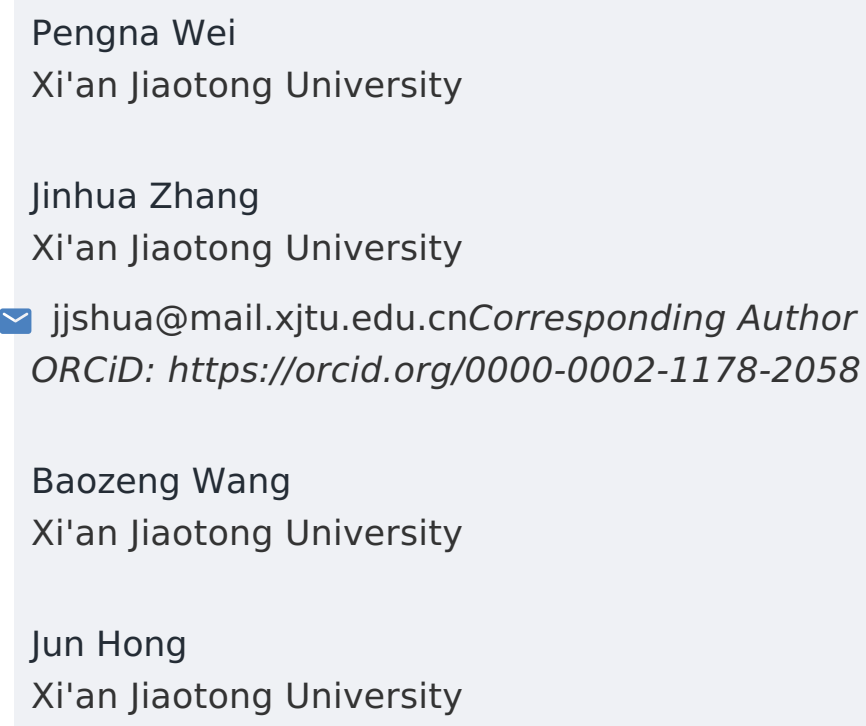

Electroencephalo gram (EEG), Surface electromyography (sEMG), Gait 50 phases, Time frequency cross mutual information (TFCMI) 
Abstract

Background: Previous researchers have found that cortex is involved in the regulation of treadmill walking. However, cortico-muscular interaction analysis in a 'fine' gait phase (such as seven phases of the gait cycle) remains lacking in the time-frequency domain. Methods: In this investigation, we used beta band electroencephalogram (EEG) data to find that eight muscle-related cortices are inconsistent at the end of the swing and stance phases. The eight muscle-related cortices differ at each phase according to gamma band EEG data. Firstly, slope sign change (SSC) and mean power frequency (MPF) features of EEG and surface electromyography (sEMG) were used to recognize the seven gait phases, which are loading response (LR), mid-stance (MST), terminal stance (TST), preswing (PSW), initial swing (ISW), mid-swing (MSW) and terminal swing (TSW). Following this, the timefrequency cross mutual information (TFCMI) method, a novel time-frequency analysis method, was applied to examine the eight muscle-related cortices in seven gait phases using beta and gamma band EEG data. Results: We firstly found that the feature set comprising SSC of EEG as well as SSC and MPF of SEMG was available for seven gait phases recognition, and secondly that TFCMI values between each sEMG channel and EEG differed significantly in the seven gait phases. Conclusions: This suggests that analysis of the seven gait phases is beneficial. These insights enrich previous findings from authors carrying out cortico-muscular interaction analysis as well as providing critical information for rehabilitation physicians.

\section{Full Text}

Due to technical limitations, full-text HTML conversion of this manuscript could not be completed. However, the manuscript can be downloaded and accessed as a PDF.

Figures 
(a)

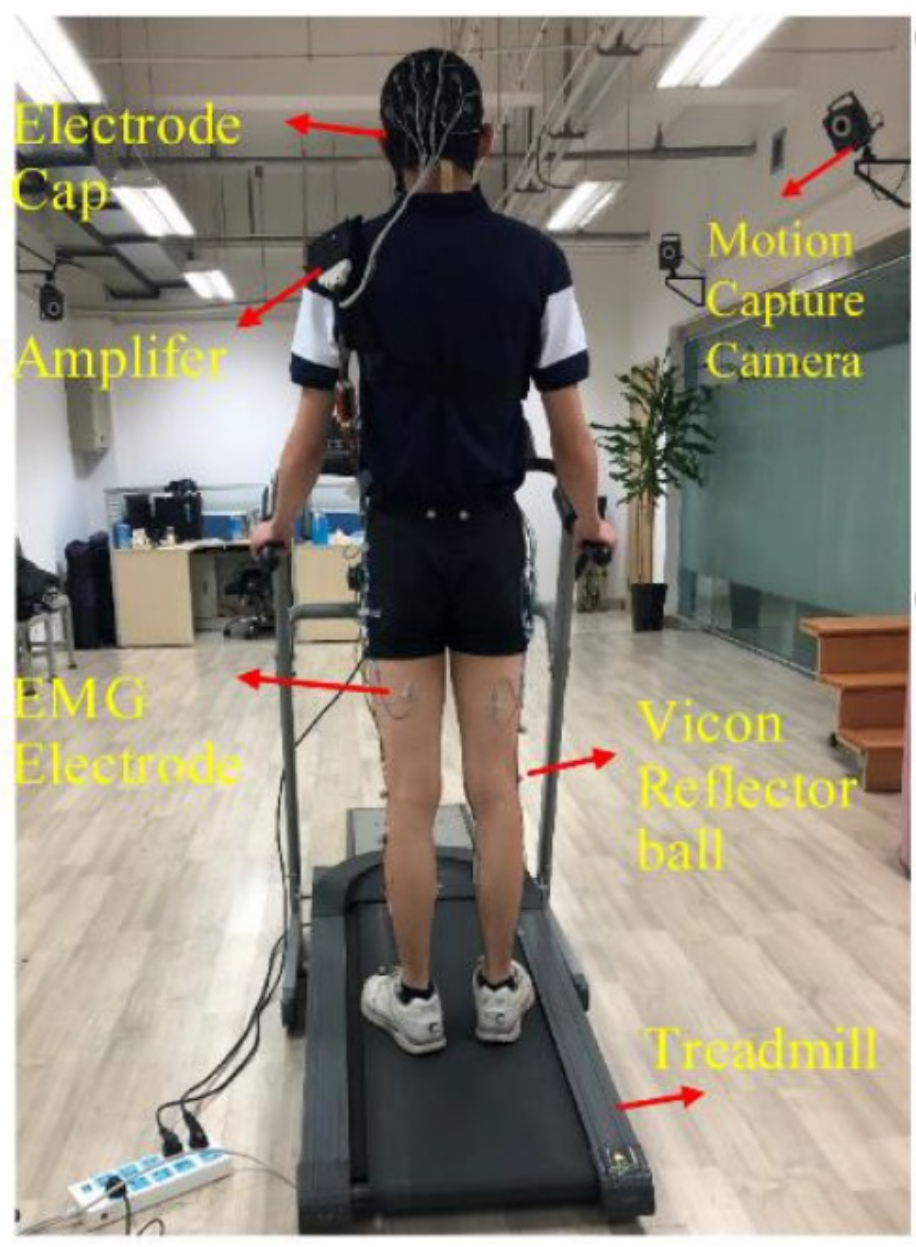

(b)

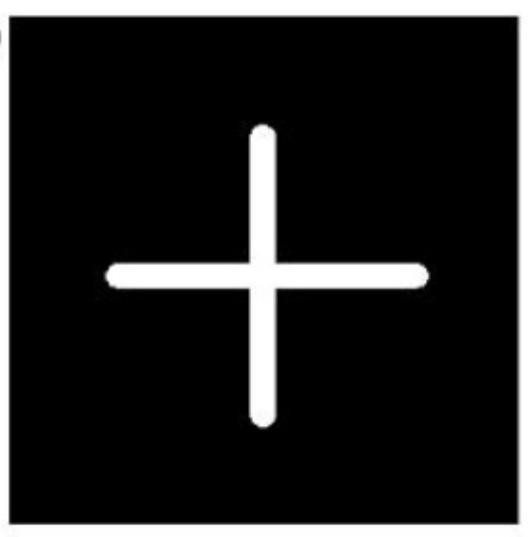

(c)

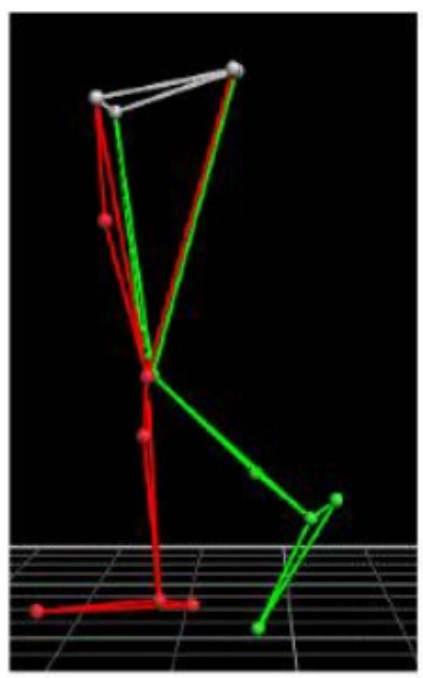

Figure 1

Experimental device, (b) cross symbol, (c) lower limb model in motion capture system . 


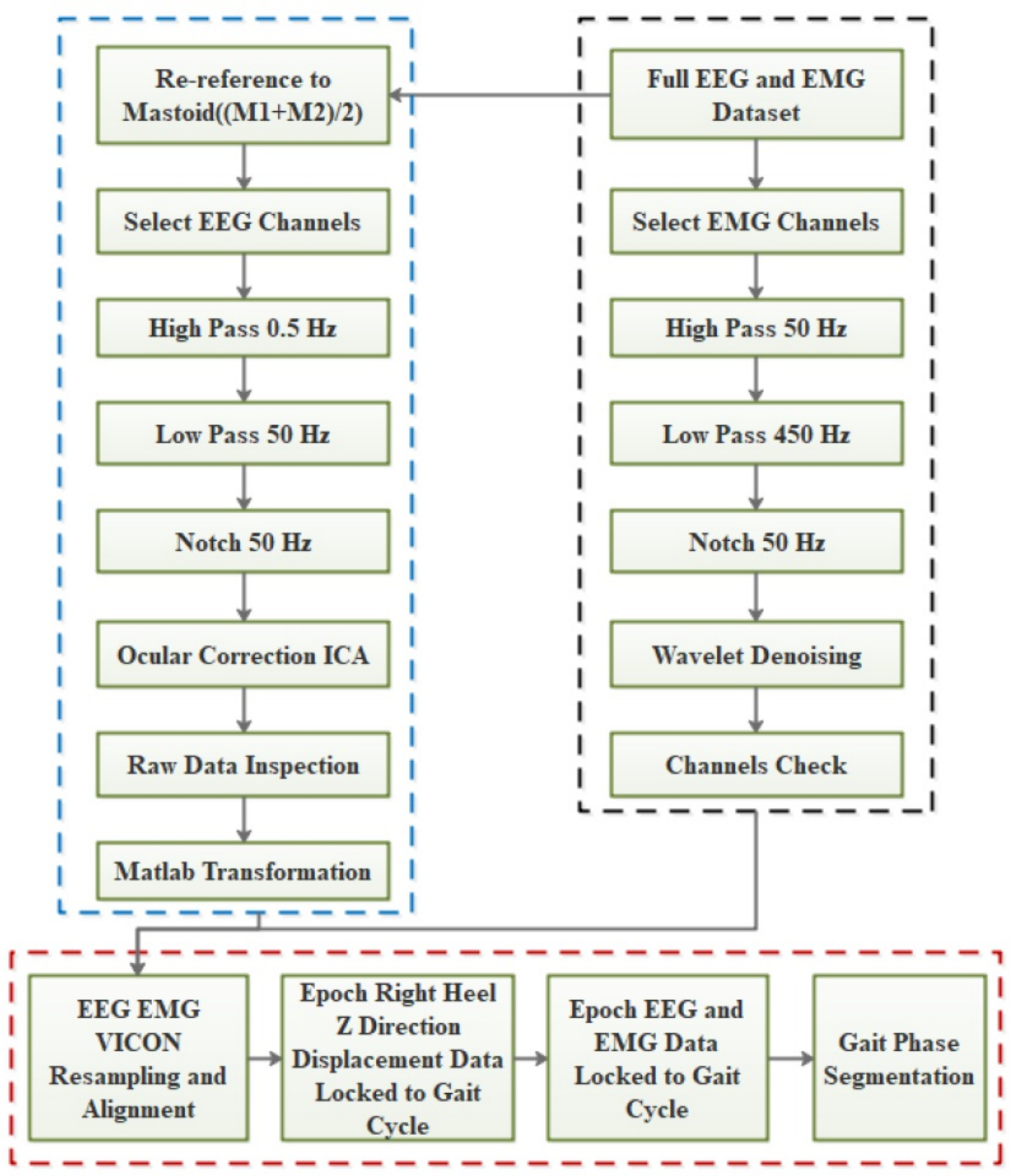

Figure 2

Outline of data processing steps. 

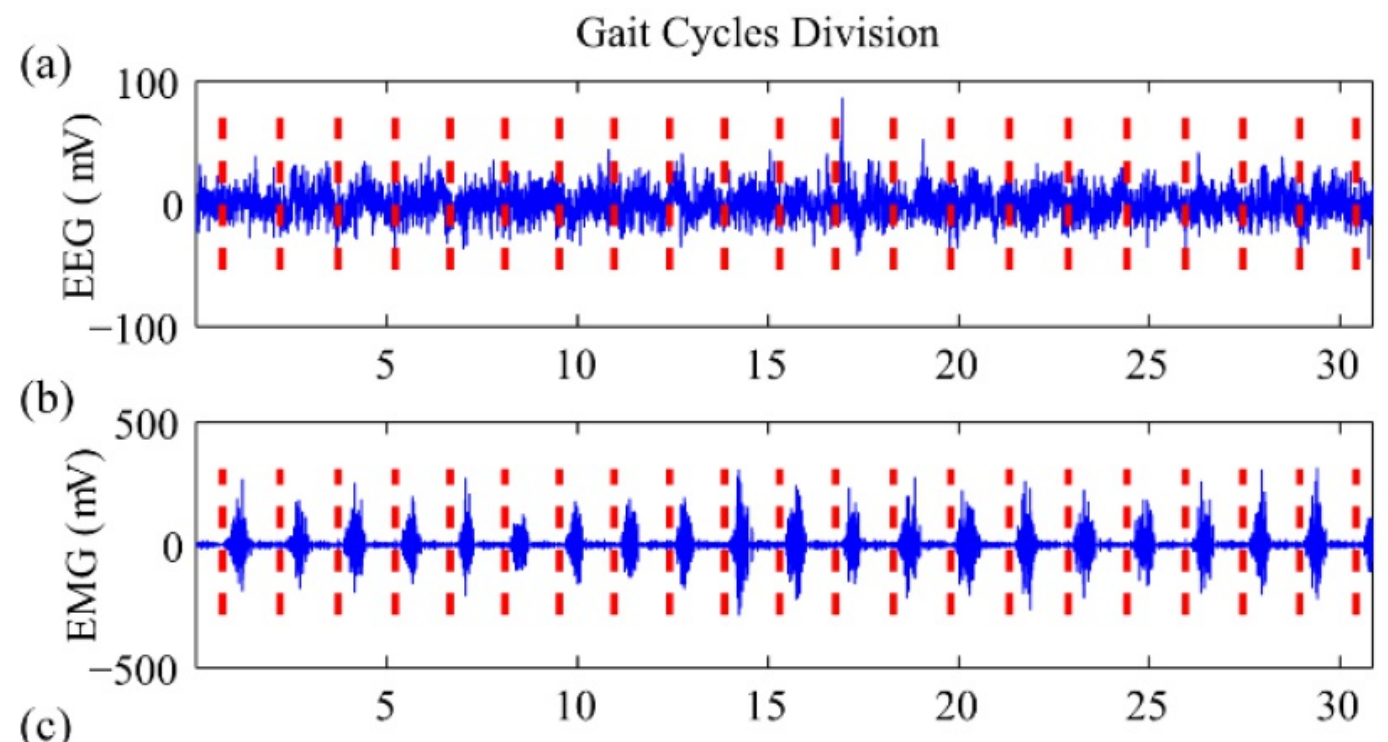

Figure 3

(a) EEG signals, (b) sEMG signals, (c) displacement in z direction of right heel. 


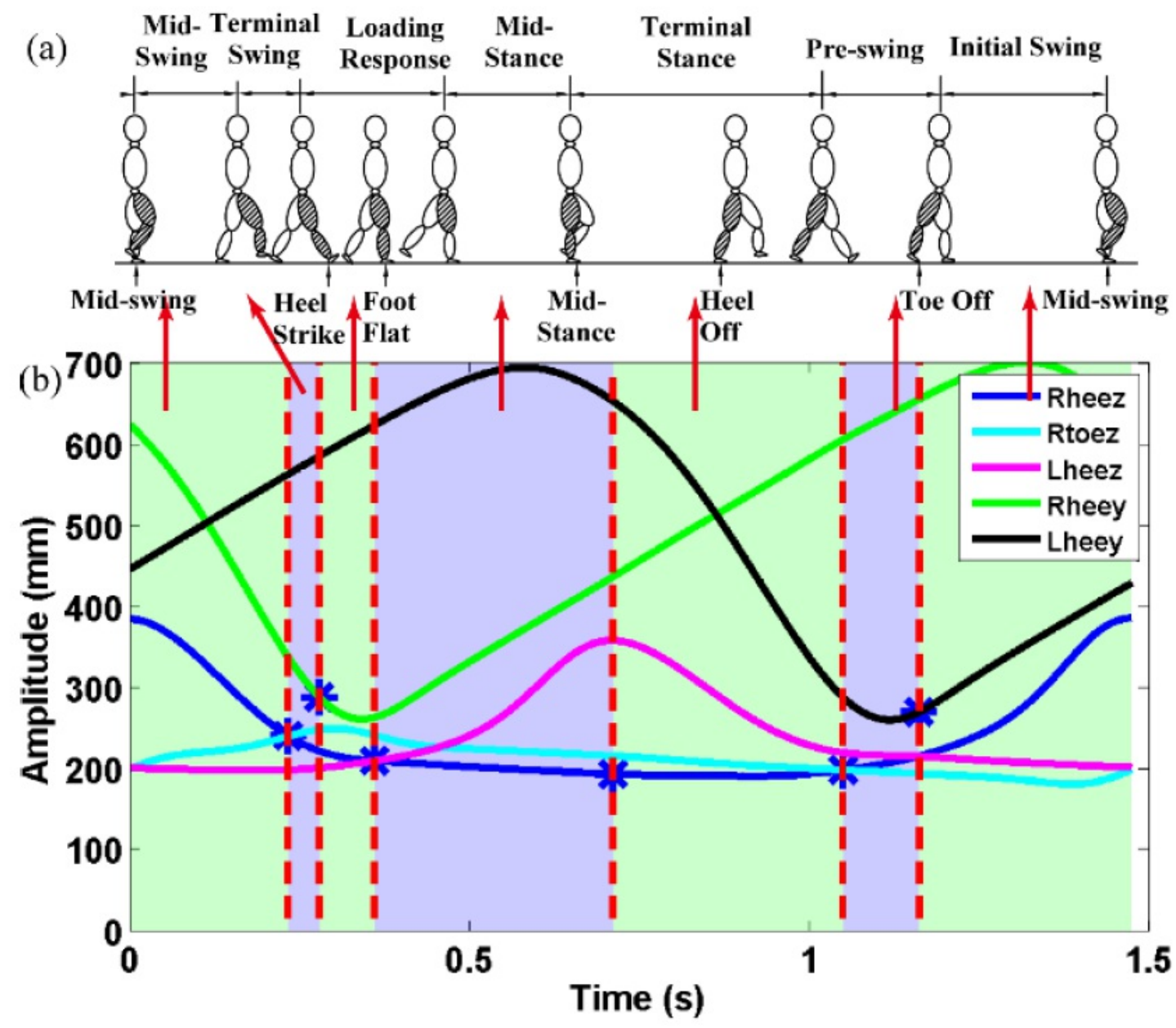

Figure 4

Gait phase division results. (a) S even gait phases of the gait cycle (b) trajectory Feature extraction 
(a)

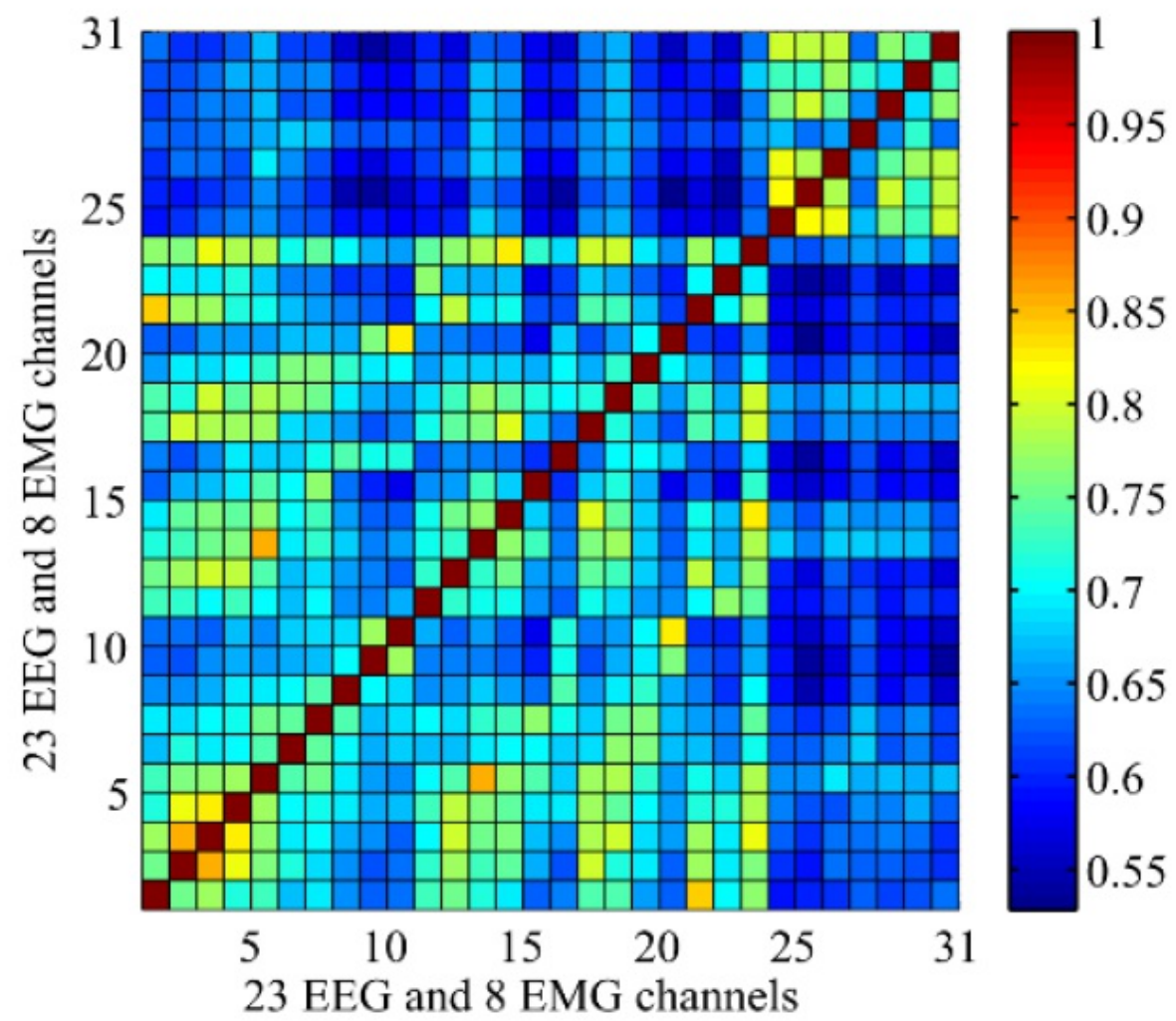

(b)

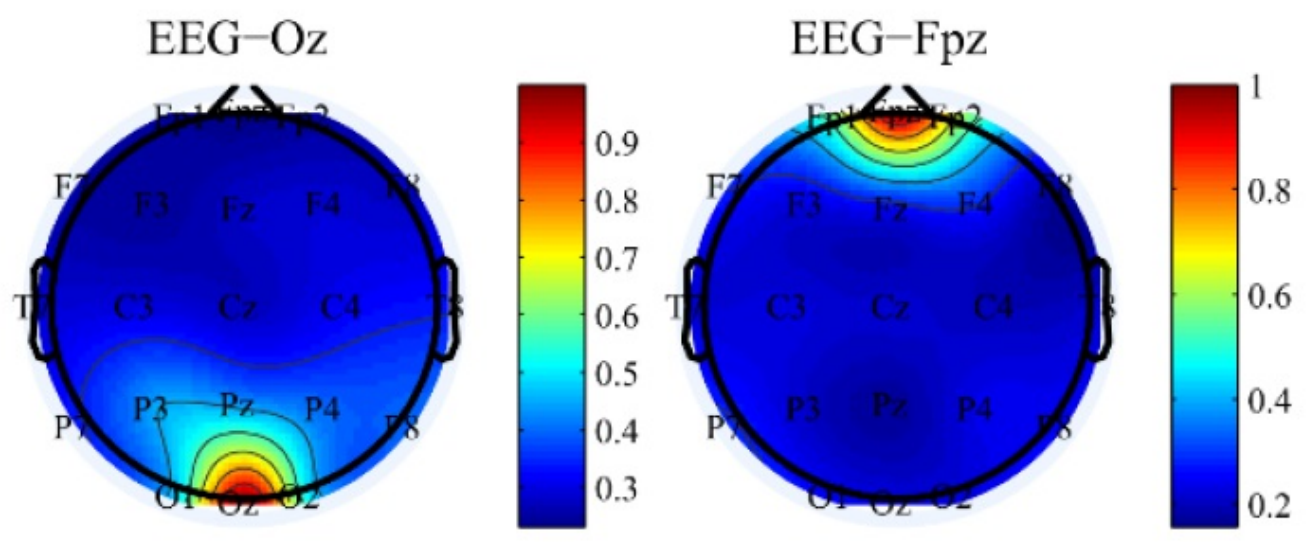

Figure 5

TFCMI computation. (a) TFCMI map, (b) topographic map of mean coupling strength from Oz channel to all EEG channels and Fpz channel to all EEG channels 

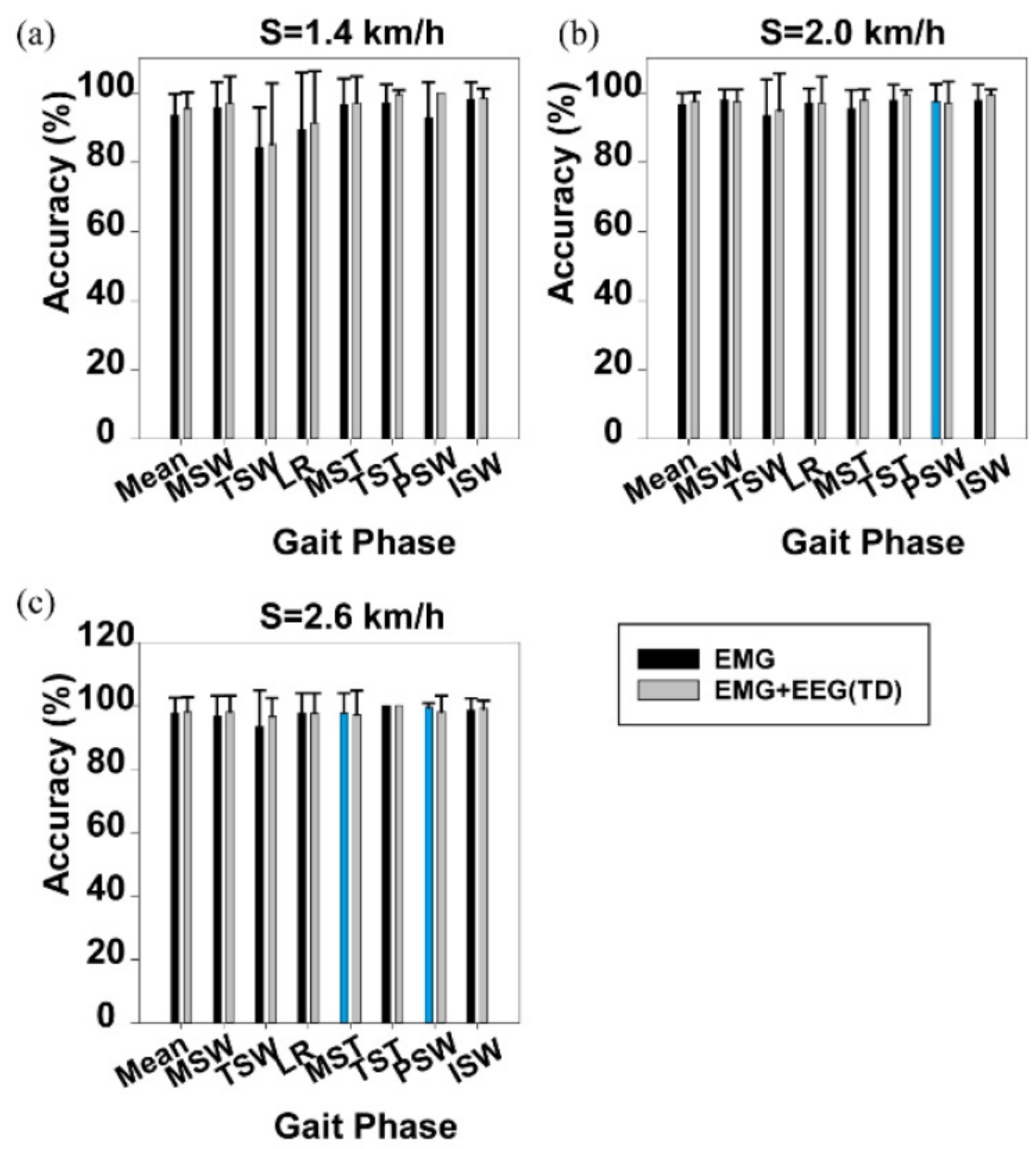

Figure 6

Gait phase recognition results based on the SSC and MPF features of sEMG as well as results based on the SSC and MPF features of SEMG and SSC feature of EEG. (a) Walking speed 1.4 $\mathrm{km} / \mathrm{h}$ (b) walking speed $2.0 \mathrm{~km} / \mathrm{h}$ (c) walking speed $2.6 \mathrm{~km} / \mathrm{h}$. The blue column indicate s that results based on S EMG features are better than SEMG features combin ed with the EEG feature of SSC. 
(a)

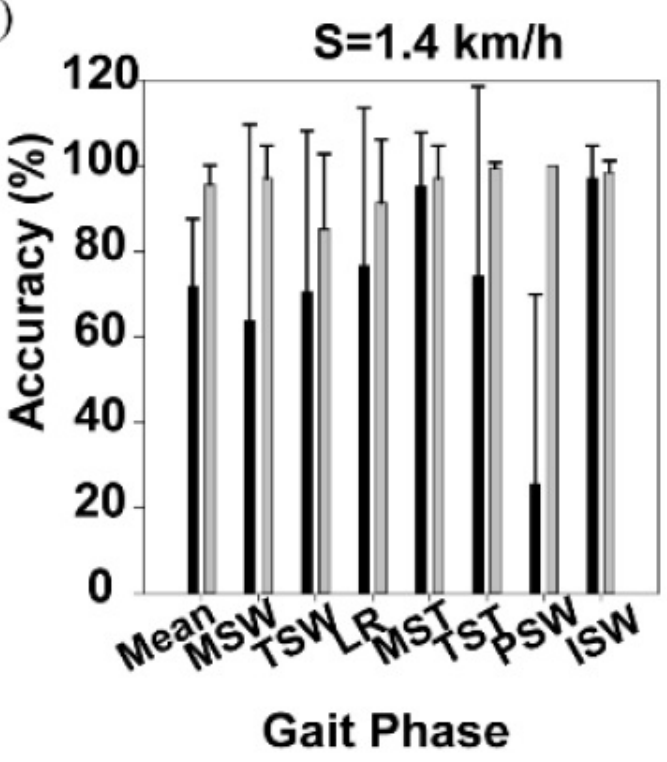

(c)

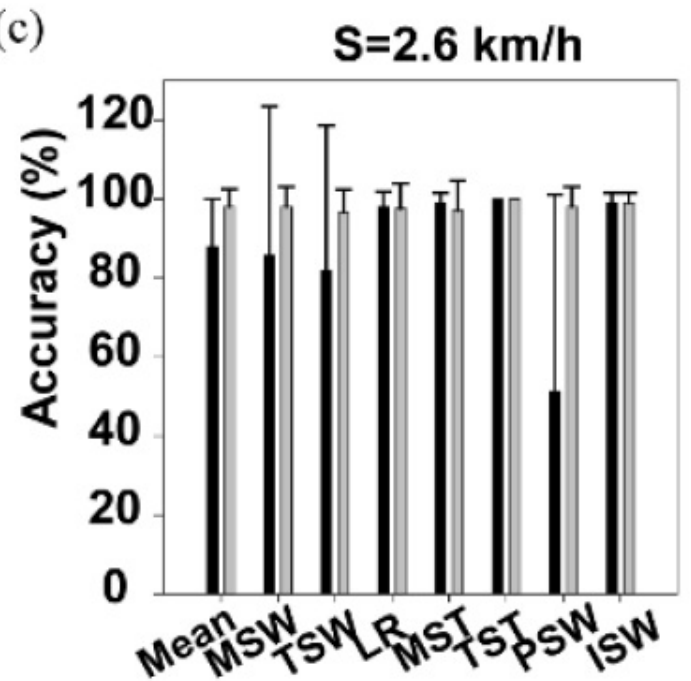

(b)

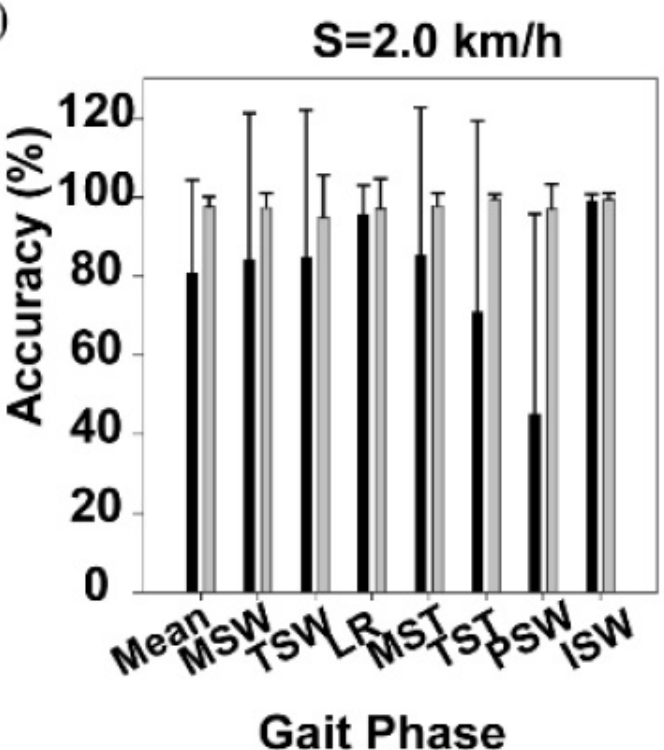

\section{Gait Phase}

Figure 7

Gait phase recognition based on the SSC and MPF of SEMG and the SSC of EEG as well as gait phase recognition based on the SSC and MPF of SEMG and the MPF of EEG. (a) Walking speed $1.4 \mathrm{~km} / \mathrm{h}$ (b) walking speed $2.0 \mathrm{~km} / \mathrm{h}$ (c) walking speed $2.6 \mathrm{~km} / \mathrm{h}$ 
LR EEG-Rt.TA EMG
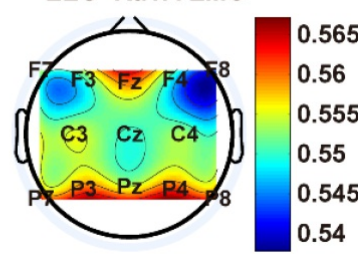

MST

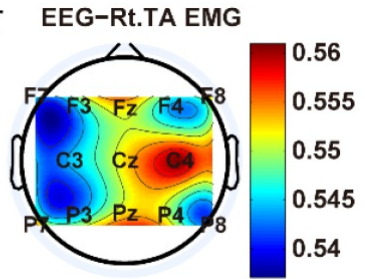

TST
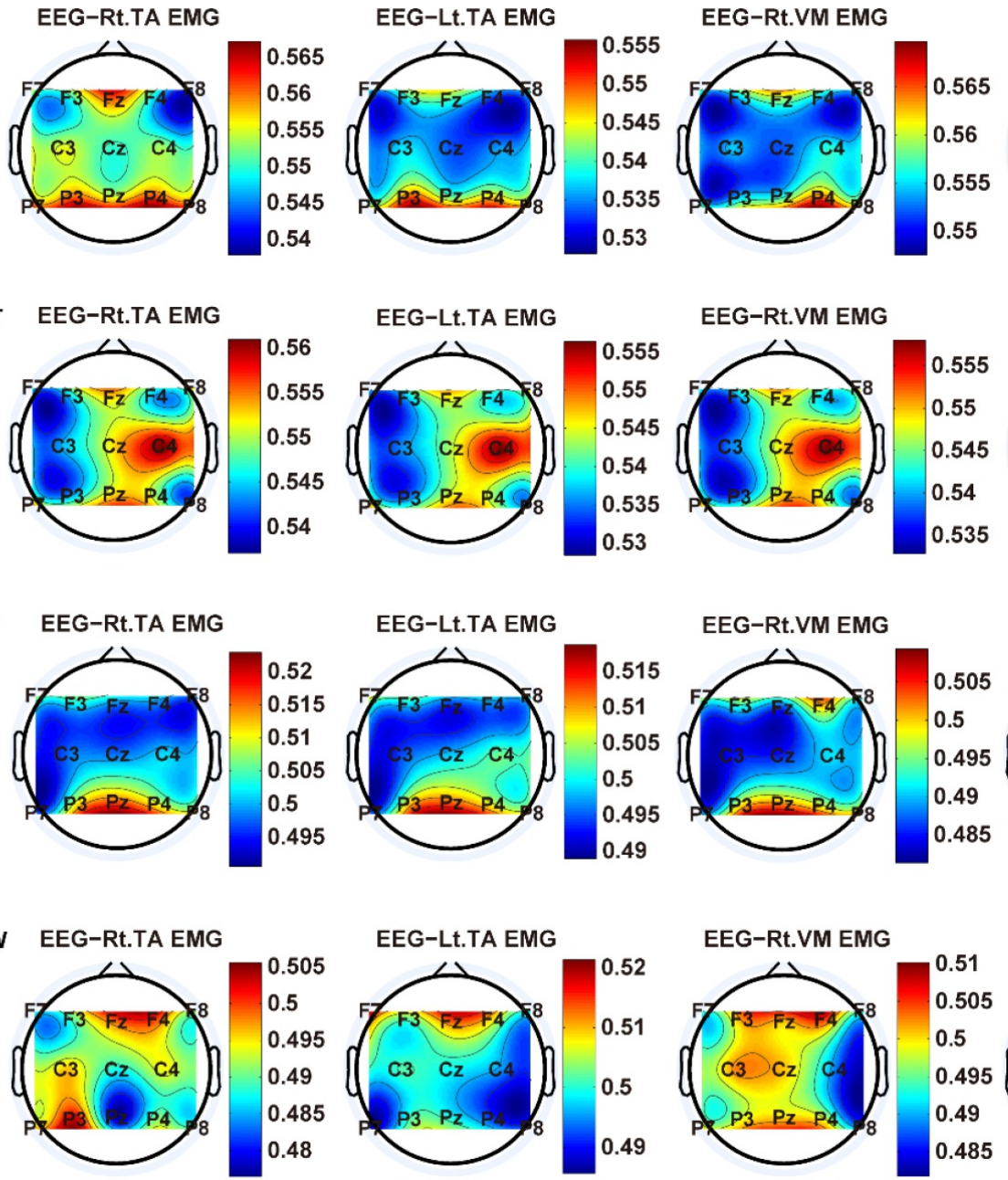

EEG-Lt.VM EMG
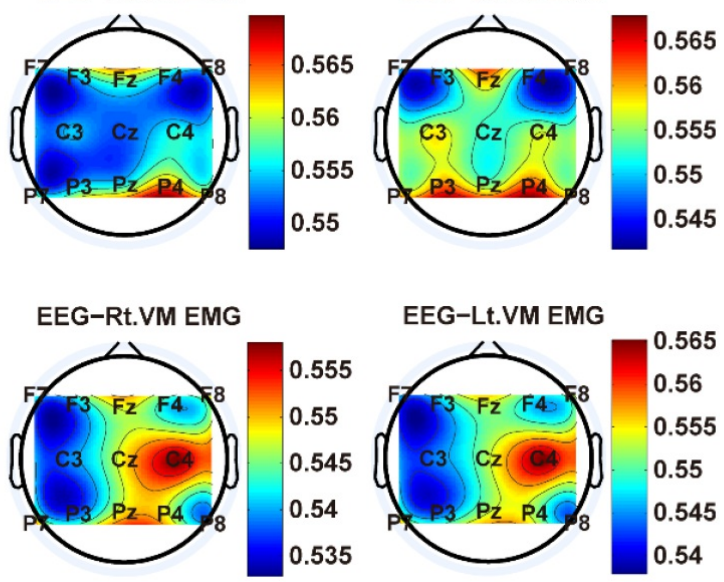

PSW EEG-RT.TA EMG
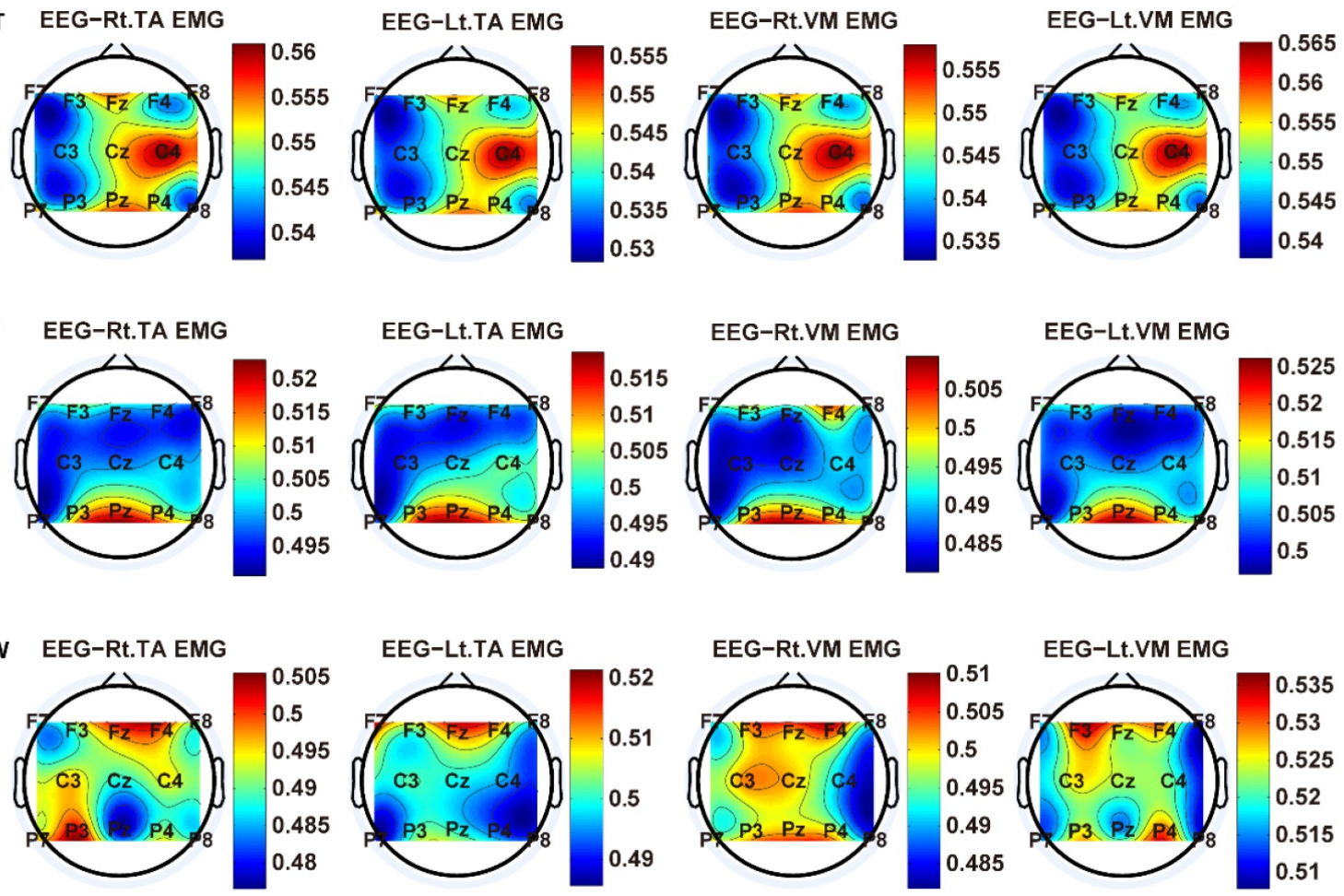

Figure 8

The TFCMI topography between EEG (beta band) and SEMG (TA, VM) of the stance phase (LR, MST, TST, PSW) PSW). 
ISW EEG-Rt.TA EMG

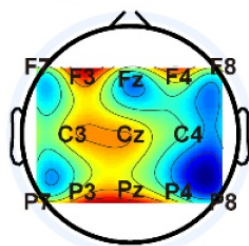

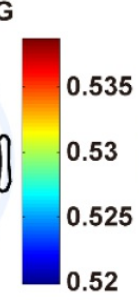

0.52
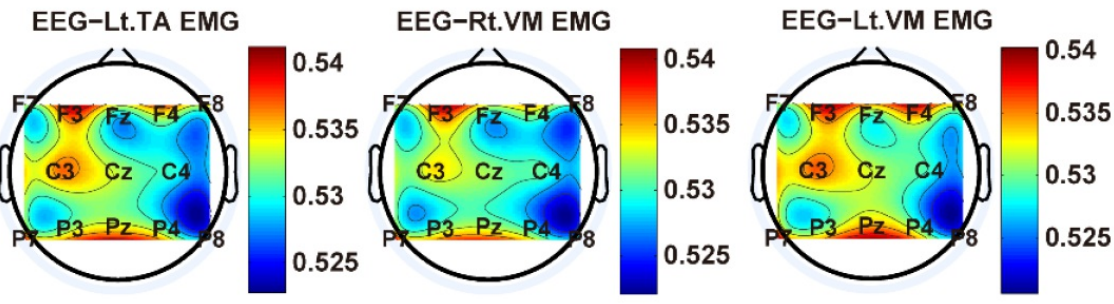

MSW EEG-RT.TA EMG
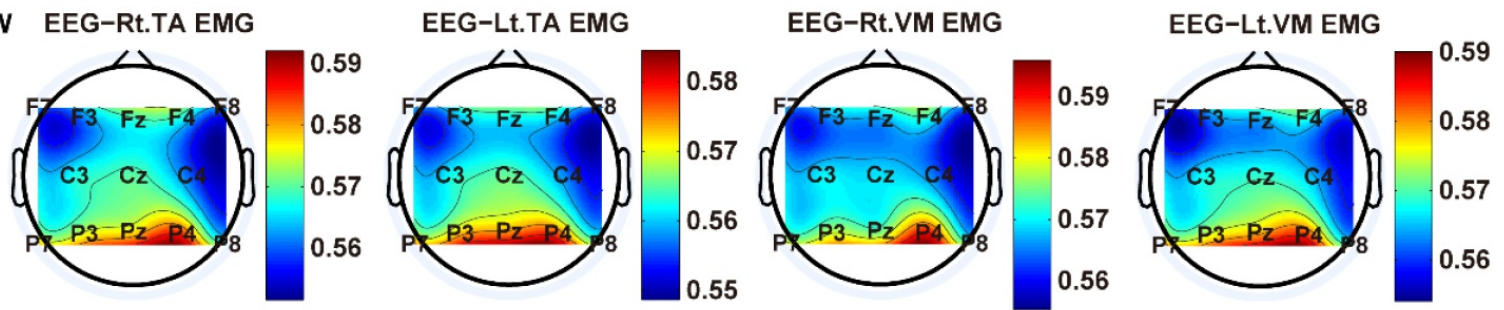

TSW EEG-Rt.TA EMG
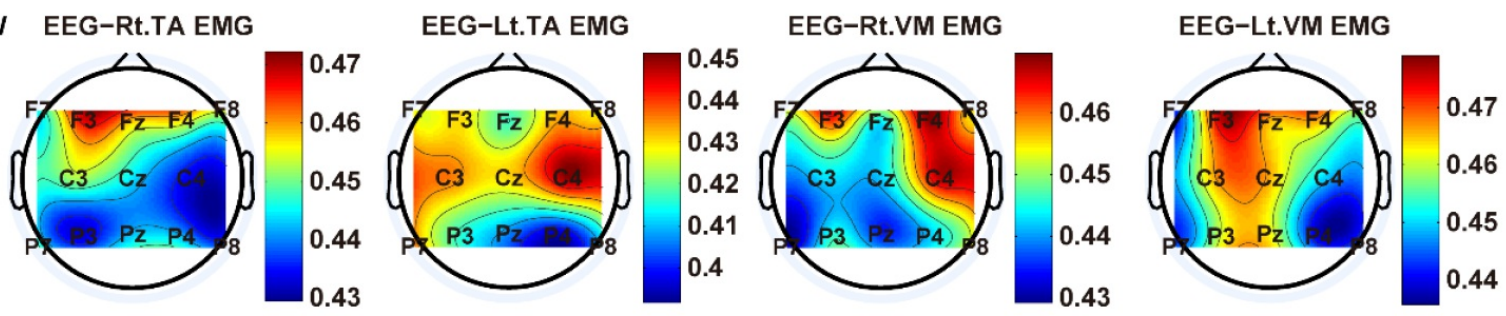

Figure 9

The TFCMI topography between EEG (beta band) and SEMG (TA, VM) of the swing phase (ISW, MSW, TSW) TSW). 
LR EEG-Rt.BF EMG
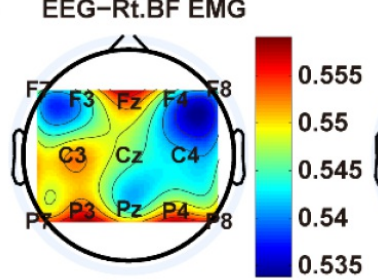

EEG-Lt.BF EMG
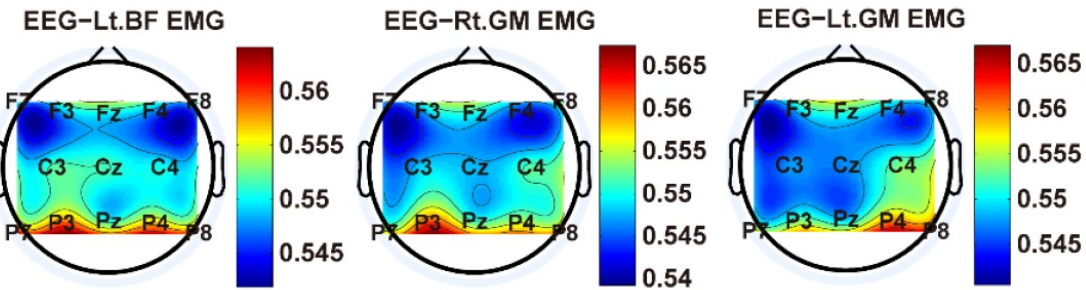

MST EEG-Rt.BF EMG
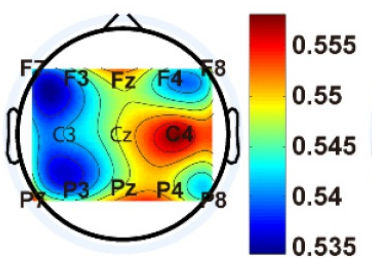

EEG-Lt.BF EMG
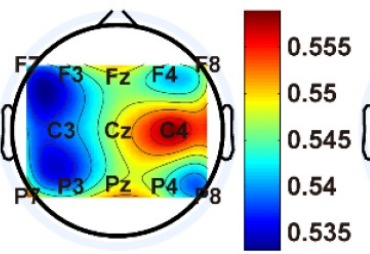

EEG-Rt.GM EMG
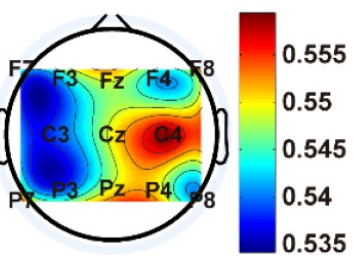

EEG-Lt.GM EMG

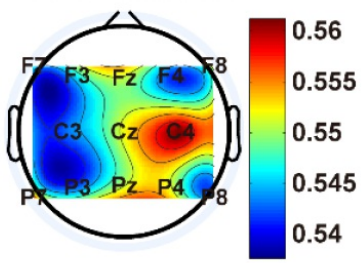

TST
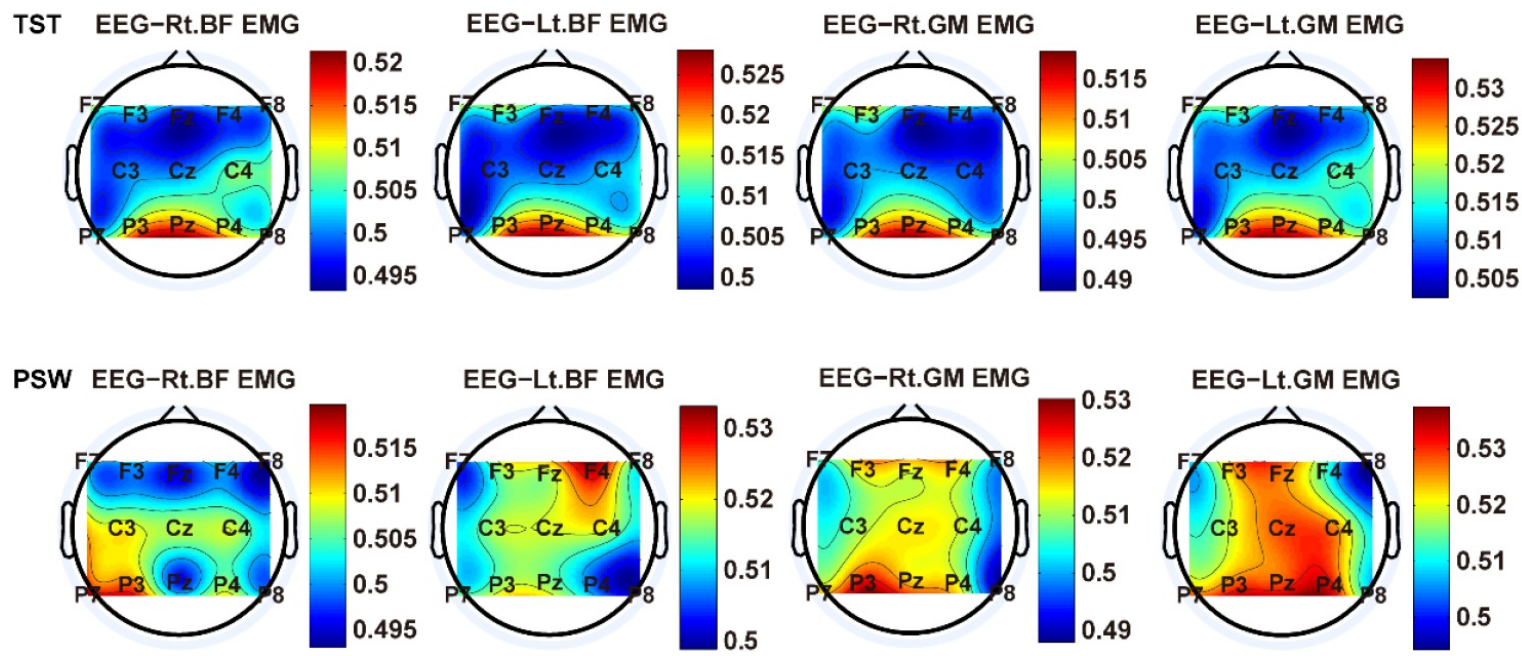

Figure 10

TFCMI topography between EEG (beta band) and SEMG (BF, GM) of the stance phase (LR,

$$
\text { MST, TST, PSW) PSW). }
$$


ISW EEG-Rt.BF EMG

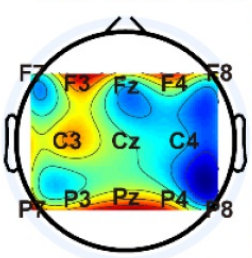

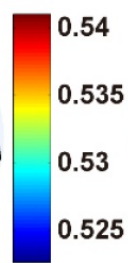

EEG-Lt.BF EMG

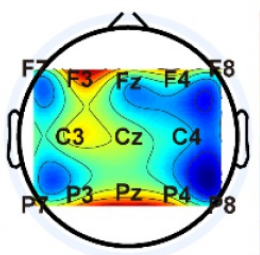

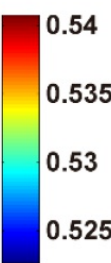

EEG-Rt.GM EMG

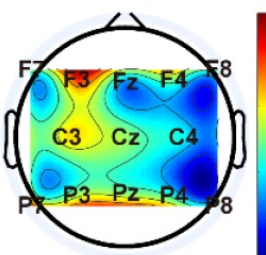

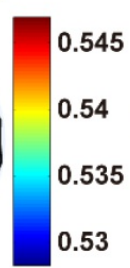

EEG-Lt.GM EMG

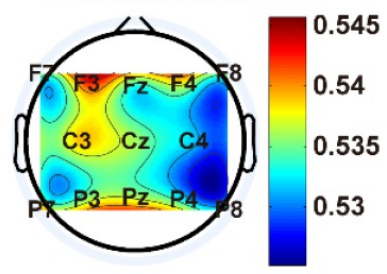

MSW EEG-Rt.BF EMG
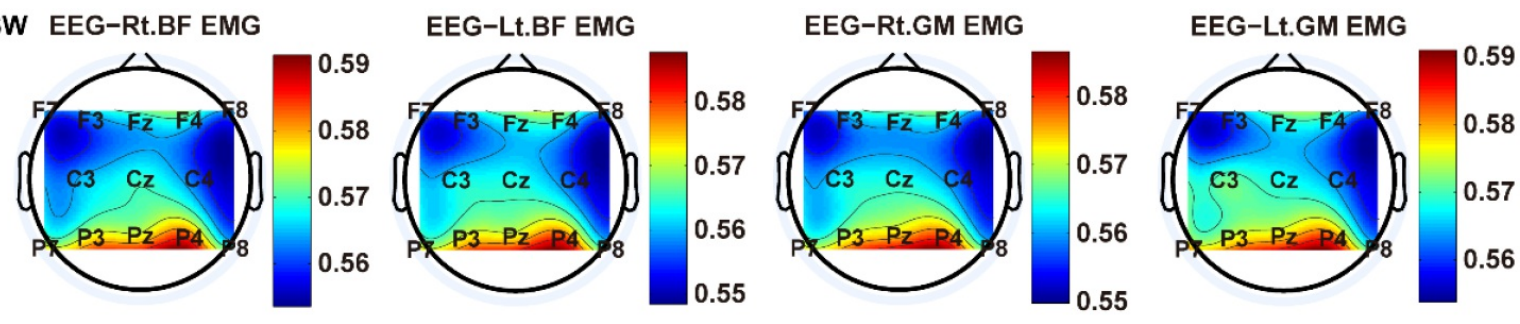

TSW EEG-Rt.BF EMG
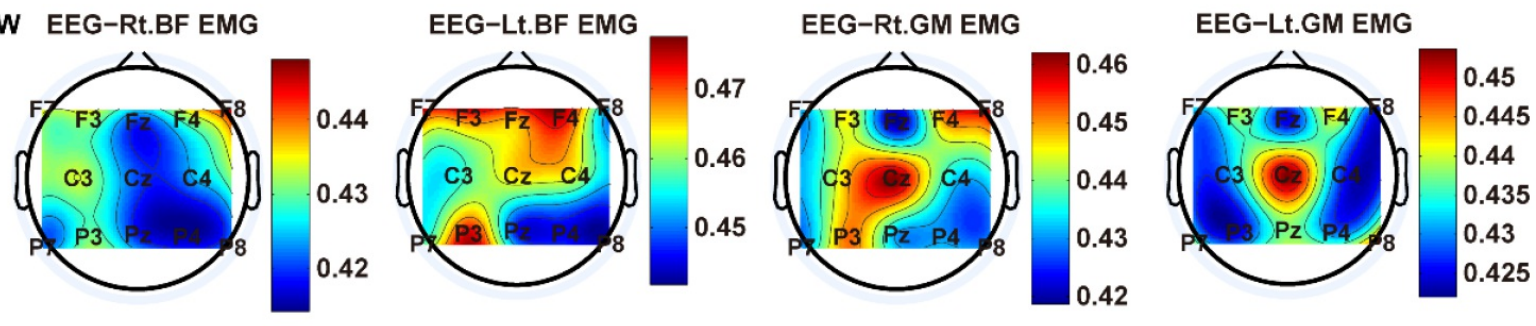

Figure 11

TFCMI topography between EEG (beta band) and SEMG (BF, GM) of the stance phase (ISW, MSW, TSW) TSW). 


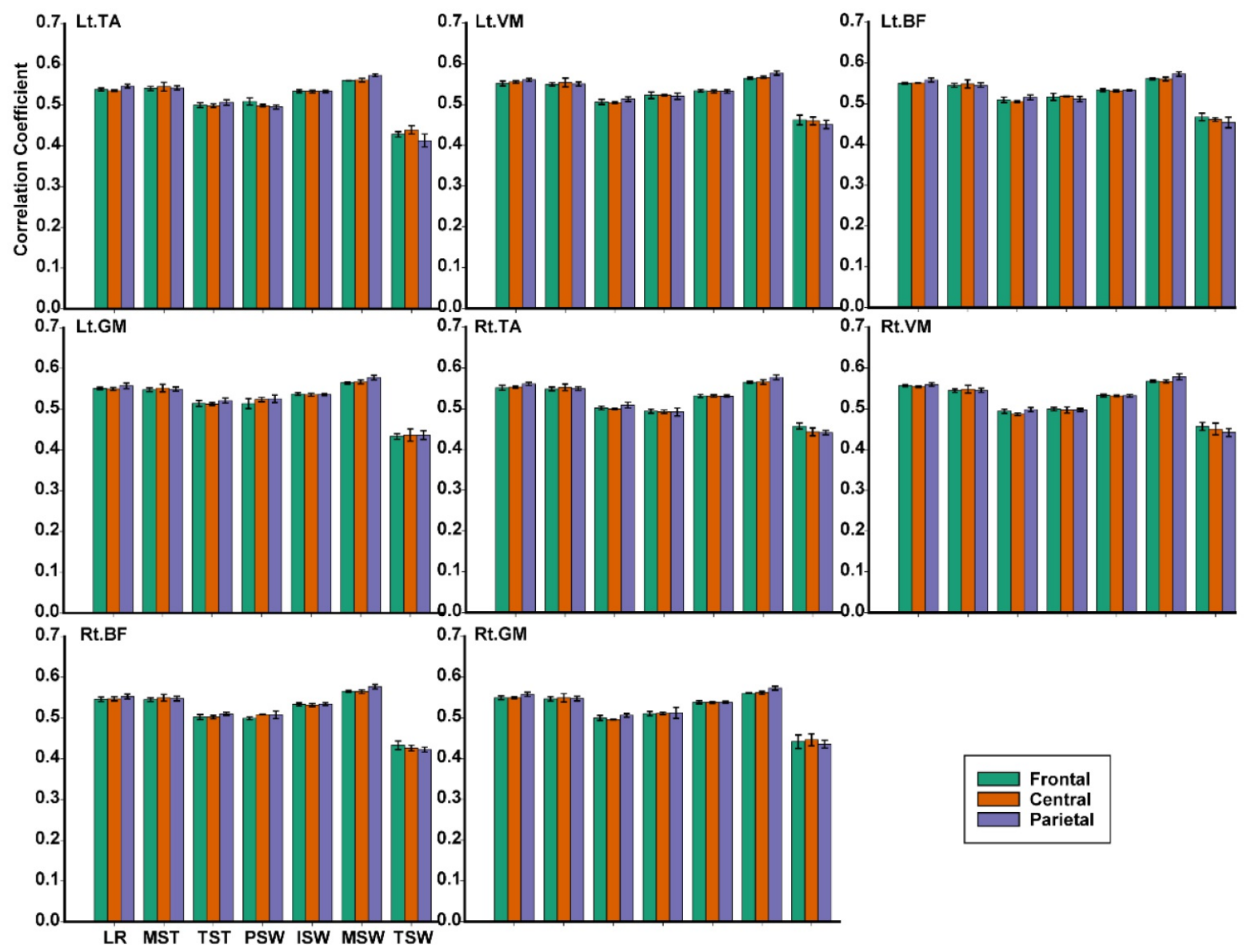

Figure 12

TFCMI values (beta band) of the frontal, central and $\mathrm{p}$ arietal lobes in the seven gait phases for the eight muscles. 

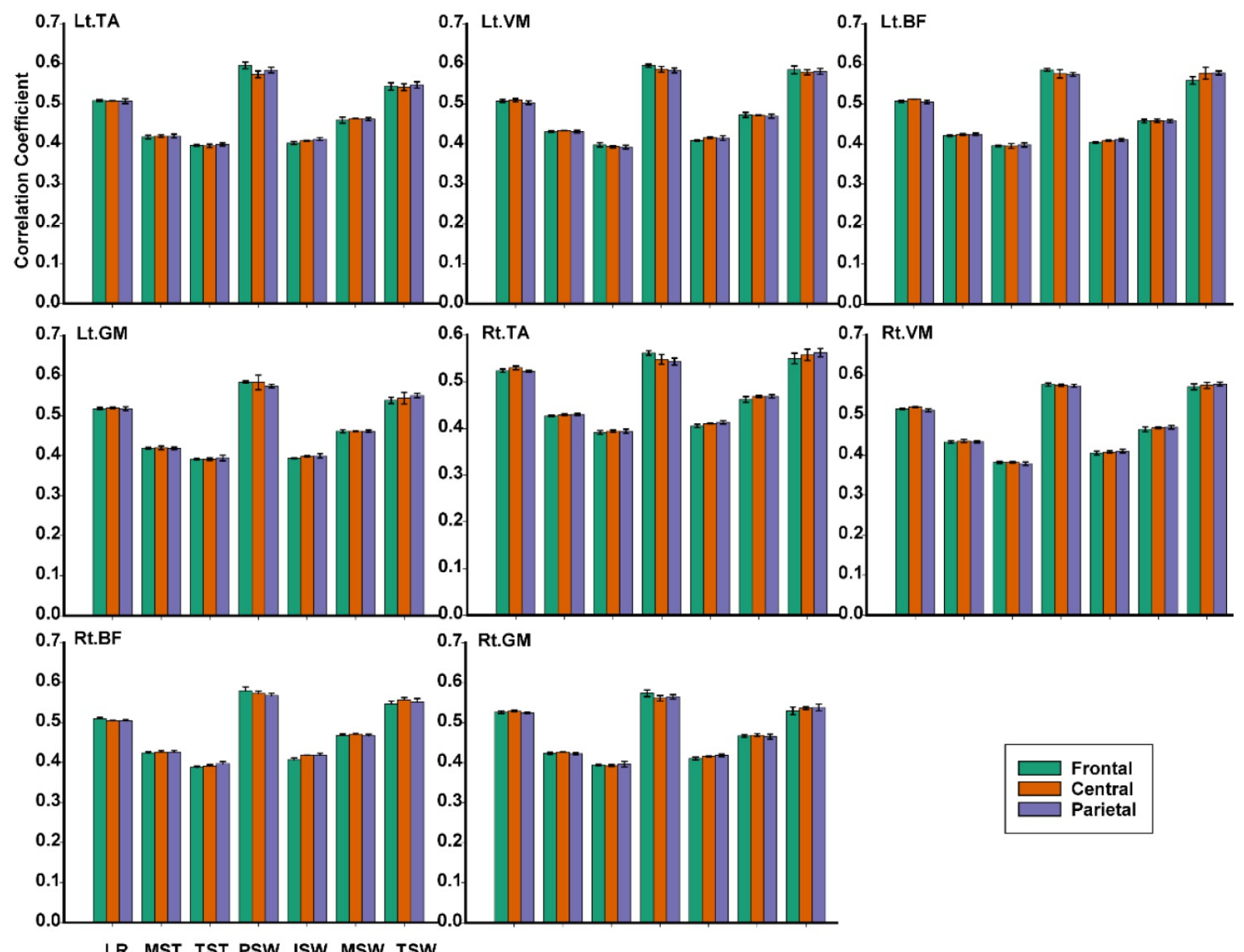

Figure 13

TFCMI values (gamma band) of the frontal, central and $p$ arietal lobes in the seven gait

phases for the eight muscles.

\section{Supplementary Files}

This is a list of supplementary files associated with this preprint. Click to download.

FigS6.tif

FigS5.tif

FigS2.tif

FigS1.tif

FigS7.tif

FigS3.tif

FigS4.tif 